\title{
EDITORIAL
}

\section{Paediatric obesity: what do we know and are we doing the right thing?}

\author{
A Pietrobelli ${ }^{1 *}$ and KS Steinbeck ${ }^{2 *}$ \\ ${ }^{1}$ Pediatric Unit, Verona University Medical School, Verona, Italy; and ${ }^{2}$ Metabolism \& Obesity Services, Department of \\ Endocrinology, Royal Prince Alfred Hospital, Camperdown, Australia
}

International Journal of Obesity (2004) 28, 2-3. doi:10.1038/sj.ijo.0802557

There is no doubt that the childhood obesity 'epidemic' is with us. Despite numerous prevalence studies, the complex relationship between the environment and the development of obesity is poorly understood in the paediatric age group. The interactions between the environment, genetic predisposition and growth in children and adolescents have not been studied as they have been in adults. Energy balance, biological function and body composition are three closely correlated entities in the study of obesity. It is often not easy to tease out cause and effect.

Both in adults and in children, the high prevalence of obesity is a concern, given the associated health risks of obesity, which in children include elevated blood pressure, hyperinsulinaemia, glucose intolerance and type II diabetes, dyslipidaemia and other early risks for cardiac disease. ${ }^{1,2}$ The child and adolescent population is not a 'mature' one. Somatic growth and development are highly relevant to the development of obesity, and boys and girls follow different growth trajectories. It is fundamental to assess this natural aspect whenever studies are carried out on a paediatric population. $^{3}$

Despite significant research into risk factors, we are as yet unable to readily detect the preobese state in children, something that would allow better targeted interventions. Parental weights, birth weight and family environments may all supply important clues. The environmental changes necessary for the prevention of obesity should closely follow intervention practices and will be advantageous for all children.

Research to identify at-risk subjects on the basis of behavioural and metabolic characteristics will be important, as will be the development of more precise techniques to detect body composition changes in children, especially the relationship between fat and fat-free mass. ${ }^{4}$ For children and

*Angelo Pietrobelli, Pediatric Unit, Verona University Medical School, Policlinic GB Rossi, Via delle Menegone 10, 37134 Verona, Italy. E-mail: angpie@tin.it adolescents, the development of better techniques to assess food intake and physical activity should be a high priority. ${ }^{5}$ Optimal methods remain undefined and methodology will vary with age.

Due both to the importance of child and adolescent obesity and to the increasing body of research in this area, the International Journal of Obesity (IJO) plans that every third volume will have a high number of paediatric articles that focus on paediatric obesity. From the manuscripts submitted to IJO, excellent papers on paediatric obesity will be selected specifically for these focus issues. In this first paediatric issue, we are pleased to have five papers that address important issues in childhood obesity - the meaning of prevalence data, the utility of weight for height indicators of adiposity, ethnicity and fatness, motor skills and obesity, and the role of the autonomic nervous system in obesity. Using the National Health and Nutrition Examination Survey (NHANES) estimated at four points in time, the work of Jolliffe examines a measure of overweight that could provide information on the extent of overweight, and not simply its presence. That is the degree to which BMI exceeds the overweight threshold, reminding us that simple prevalence data do not tell us all we need to know. The results indicate that there have been large shifts in the BMI distribution of overweight children that the simple prevalence of overweight does not reveal. The rapidity of this change to the right of the overweight spectrum is a recent phenomenon in children.

Freedman et al have utilised data on 11406 children and 2911 adults from the Bogalusa Heart Study to explore the relationship between childhood height to BMI, skinfold thicknesses and adult obesity, together with the relationship of different childhood weight-height indices to adult adiposity. Cross-sectional analysis examined the relation of height to adiposity (measured by BMI and skinfold thickness) and fasting insulin levels, and longitudinal analysis examined the relation of childhood height and weightheight indices to adult levels of adiposity and fasting 
qinsulin. No weight-height index was able to clearly distinguish excess weight due to adiposity, and the use of BMI results in a high prevalence of overweight among taller children. The utility of simple field measures as surrogates of adiposity is accepted, but these do not reveal the whole story in children.

Moore et al's study considered leptin, its function in regulating energy balance and fat stores and addressed ethnic differences. Using two cohorts from Italy and Gambia, the results showed a striking difference in both fatness and leptin levels between the two populations. These findings have implications for the use of growth reference charts and cutoff points for levels of adiposity in different ethnic groups.

The study by Graf et al examined the association between BMI and different aspects of energy expenditure-gross motor development, endurance performance and leisure habits by questionnaire - in 668 first-grade children. This paper addresses, for the first time, the correlation between early childhood overweight and gross motor development, and demonstrated a correlation between increased BMI and poorer results in both gross motor development and endurance performance. In addition, active leisure behaviors in children were associated with more advanced gross motor development, whereas sedentary habits (including television viewing) correlated with poorer gross motor development. These findings support the early enhancement of gross motor skills and raise questions about cause and effect in the development of early childhood obesity.

Finally, Nagai and Moritani investigated the autonomic nervous system, a key component in energy balance and fat storage regulation and a potential aetiological factor in childhood obesity, and correlated its activity in lean and obese subjects. Differences in physical activity levels were also considered. Using a two-arm design, obese active/ inactive $v s$ lean active/inactive, there were two important results: (1) a reduced sympathetic as well as parasympathetic nervous activity in obese subjects compared with lean subjects was identified; and (2) the finding that regular sports activity contributed to enhancement of the overall autonomic nervous system activity in both lean and obese children.

These five paediatric studies are well attuned to research priorities in childhood obesity. It is important to define the particular health risks (both current and future) in children as these relate to increasing BMI, reduced physical activity and dietary habits. ${ }^{6}$
It is clear that childhood obesity must be addressed at the whole of community level. The evidence base, which has until recently been limited in children and adolescents, is rapidly expanding. Research strengthens clinical management, and clinicians need to be attuned to the various factors that can influence normal growth and that cause deviation towards less healthy growth. Energy intake and diet composition, food preferences, physical activity and sedentary behaviours, nutritional and hormonal status, and the influence of behavioural factors all play a role in body fatness. The challenge is the more precise measurement of such factors, including body composition and energy expenditure, and longitudinal studies during childhood growth and development that can address consequences of excess fatness both in childhood and into adult life.

Finally, the community needs to be engaged in the prevention and early intervention of childhood obesity, as obesity cannot be managed by health professionals alone. Different community sectors and influential bodies need to drive macroenvironmental change. Parents need to be aware of their fundamental role in altering the microenvironment. Schools have a responsibility for providing healthy food choices and adequate time spent in physical activity. ${ }^{6,7}$ More research is needed not only to identify children at risk for obesity, but to test the prevention strategies that have been advanced as potential solutions. The IJO will continue to record the advancements in the knowledge around childhood obesity.

\section{References}

1 World Health Organization. Obesity: preventing and managing the global epidemic, World Health Organization Technical Report Series No. 894. World Health Organization: Geneva, Switzerland; 2000.

2 Dietz WH, Bland MG, Gortmaker SL, Molloy M, Schmid TL. Policy tools for the childhood obesity epidemic. J Law Med Ethics 2002; 30: 83-87.

3 Forbes GB. Human body composition: growth, aging and activity. Springer Verlag: New York; 1987.

4 Pietrobelli A, Heymsfield SB. Establishing body composition in obesity. J Endocrinol Invest 2002; 25: 884-892.

5 Steinbeck KS. Obesity: the science behind the management. Intern Med J 2002; 32: 237-241.

6 Krebs NF, Jacobson MS, American Academy of Pediatrics Committee on Nutrition. Pediatrics 2003; 112: 424-430.

7 Hill JO, Trowbridge FL. The causes and health consequences of obesity in children and adolescents. Pediatrics 1998; 101: 497-574. 